\title{
Quark Wigner Distributions and Orbital Angular Momentum in Light-front Dressed Quark Model
}

\author{
Asmita Mukherjee, Sreeraj Nair and Vikash Kumar Ojha \\ Department of Physics, \\ Indian Institute of Technology Bombay, \\ Powai, Mumbai 400076, India.
}

(Dated: October 23, 2018)

\begin{abstract}
We calculate the Wigner functions for a quark target dressed with a gluon at one loop in perturbation theory. The Wigner distributions give a combined position and momentum space information of the quark distributions and are related to both generalized parton distributions (GPDs) and transverse momentum dependent parton distributions (TMDs). We calculate and compare the different definitions of quark orbital angular momentum and the spin-orbit correlations in this perturbative model. We compare our results with other model calculations.
\end{abstract}




\section{INTRODUCTION}

In classical physics, a system of particles can be described in terms of phase space distributions, which represent the density of particles at a point in the phase space at a given time. In quantum mechanics, position and momentum operators do not commute and they cannot be determined simultaneously. Thus in quantum mechanics one cannot define phase space distributions. Wigner distributions in quantum mechanics have been introduced long ago [1], which can be thought of as quantum mechanical phase space distributions, however it cannot be interpreted as probability distribution for the reason above and is not positive definite. Wigner distributions become classical phase space distributions in the limit $h \rightarrow 0$. A quantum mechanical Wigner distribution for the quarks and gluons in the rest frame of the nucleon was introduced in [2, 3. Reduced Wigner functions are obtained from the seven dimensional most general Wigner distributions by integrating the minus component of the momentum. Reduced Wigner distributions are functions of three position and three momentum variables and as discussed above are not measurable. To obtain measurable quantities one has to integrate over more variables. Integrating out the momentum variables one can relate the reduced Wigner distributions to generalized parton distributions (GPDs) and integrating out the position variables one gets the transverse momentum dependent parton distributions (TMDs). Thus the Wigner distributions can be thought of as more general mother distributions in which both position and momentum space information of quarks and gluons are encoded.

Wigner distributions are related to the generalized transverse momentum dependent correlation functions (GPCFs) [4, 5] of the nucleon, which are the fully unintegrated, off-diagonal quark-quark correlators. An overlap representation for the above using model light-front wave functions has been studied in [6]. If one integrates over the minus component of the momentum (light-cone energy) one gets the generalized transverse momentum dependent parton distributions (GTMDs). These are functions of the 3-momentum of the quark and the momentum transfer to the nucleon $\Delta_{\mu}$. In [7] the authors introduced five dimensional Wigner distributions in infinite momentum frame by integrating the GTMDs over the momentum transfer in the transverse direction $\Delta_{\perp}$. These Wigner distributions are functions of the two position and three momentum variables. Working in infinite momentum frame, or equivalently using the light- 
cone formalism has several advantages as the transverse boosts are Galilean, or do not involve dynamics, and longitudinal boost is just a scale transformation [8]. So it is easier to have an intuitive picture of the parton distributions in the nucleon. As discussed before, Wigner distributions do not have probabilistic interpretation due to uncertainty principle. Integrating out one or more variables one can define new distributions that have probabilistic interpretation. Depending on whether the nucleon and the quark is polarized or unpolarized several such distributions can be defined. In this work we shall restrict ourselves to longitudinal polarization's only. As Wigner distributions cannot be measured, model calculations are of importance to understand what kind of information about the quark-gluon correlation in the nucleon can be obtained from them as well as to verify to what extent different model dependent and model independent relations among various distributions are satisfied. However, integrating out more variables gives measurable quantities having the interpretation of probability densities. In [7] the Wigner distributions for quarks and gluons have been studied in light cone constituent quark model and in light-cone chiral quark soliton model. Both these models have no gluonic degrees of freedom and the Wilson line becomes unity.

Quark orbital angular momentum (OAM) contribution to the total spin of the nucleon has gained considerable attention since the EMC experiments [9] which showed that the quark intrinsic spin contribution was less than expected. Also recent polarized beam experiments suggest that the gluon polarization contribution to the total spin of the proton is very small. Wigner distributions are related to the OAM carried by the quarks in the nucleon. As suggested from the experimental data, a substantial part of the spin of the nucleon comes from quark and gluon OAM. The issue of gauge invariance and experimental measurability of the OAM contribution complicates the issue of a full understanding of such contributions [10]. Theoretically there exist mainly two definitions of OAM : one obtained from the sum rules of GPDs and the other, canonical OAM distribution in the light cone gauge. It has been shown in the literature that these two different distributions are projections of Wigner distributions with different choice of gauge links and they are related by a gauge dependent potential term [11-13]. In [14, 15] the canonical OAM in light-front gauge is shown to be related to the twist three GPDs.

In this paper, we present a calculation of the quark Wigner distributions in light-front 
Hamiltonian formulation using overlaps of light-front wave functions (LFWFs). This approach is based on [16]. This has the advantage that it gives an intuitive picture of deep inelastic scattering (DIS) processes in field theory while keeping close contact with parton model, but the partons are now field theoretic partons, they are non-collinear, massive and also interacting [17]. However, they are still on-mass shell. An expansion of the target state in Fock space in terms of multi-parton LFWFs allows one to calculate the matrix elements of operators. The nonperturbative light-front wave functions are boost invariant. While the non-perturbative LFWFs for a bound state like the nucleon requires a model light-front Hamiltonian, it is interesting and useful to replace the bound state by a simple composite two-body spin $1 / 2$ state, like a quark at one loop in perturbation theory. This is a relativistic state and the relativistic two-parton LFWFs can be calculated analytically in light-front Hamiltonian perturbation theory. These wave function is a function of the mass of the quark. It mimics the LFWF of a two-particle bound state [18]. In this work we calculate the Wigner distributions and OAM for a quark dressed with a gluon in light-front Hamiltonian approach. We follow the formalism of [16] where it was shown that in light-front gauge one can write the light-front QCD Hamiltonian entirely in terms of the dynamical degrees of freedom and using a certain representation of the Dirac gamma matrices, it is possible to write the theory in terms of two-component fermion spinors and transverse components of the gauge field. This two-component approach has been used successfully to investigate the GPDs. Here we use this formalism to investigate the Wigner distributions.

The plan of the paper is as follows. In section II we calculate the Wigner distributions for a dressed quark. In section III we calculate the OAM in the same model. We present the numerical results in section IV and conclusions in section $\mathrm{V}$.

\section{WIGNER DISTRIBUTIONS}

The Wigner distribution of quarks can be defined as the two-dimensional Fourier transforms of the generalized transverse momentum distributions (GTMDs) [4, 7]

$$
\rho^{[\Gamma]}\left(b_{\perp}, k_{\perp}, x, \sigma\right)=\int \frac{d^{2} \Delta_{\perp}}{(2 \pi)^{2}} e^{-i \Delta_{\perp} . b_{\perp}} W^{[\Gamma]}\left(\Delta_{\perp}, k_{\perp}, x, \sigma\right)
$$


where $\Delta_{\perp}$ is momentum transfer of dressed quark in transverse direction and $b_{\perp}$ is 2 dimensional vector in impact parameter space conjugate to $\Delta_{\perp} \cdot W^{[\Gamma]}$ is the quark-quark correlator given by

$$
\begin{array}{r}
W^{[\Gamma]}\left(\Delta_{\perp}, k_{\perp}, x, \sigma\right)=\left\langle p^{+}, \frac{\Delta_{\perp}}{2}, \sigma\left|W^{[\Gamma]}\left(0_{\perp}, k_{\perp}, x\right)\right| p^{+},-\frac{\Delta_{\perp}}{2}, \sigma\right\rangle \\
=\left.\frac{1}{2} \int \frac{d z^{-} d^{2} z_{\perp}}{(2 \pi)^{3}} e^{i\left(x p^{+} z^{-} / 2-k_{\perp} . z_{\perp}\right)}\left\langle p^{+}, \frac{\Delta_{\perp}}{2}, \sigma\left|\bar{\psi}\left(-\frac{z}{2}\right) \Omega \Gamma \psi\left(\frac{z}{2}\right)\right| p^{+},-\frac{\Delta_{\perp}}{2}, \sigma\right\rangle\right|_{z^{+}=0} .
\end{array}
$$

We define the initial and final dressed quark state in the symmetric frame [19] where $p^{+}$and $\sigma$ defines the longitudinal momentum of the target state and its helicity respectively. $x=k^{+} / p^{+}$ is the fraction of longitudinal momentum of the dressed quark carried by the quark. In the symmetric frame the transverse momentum transfer $\left(\Delta_{\perp}\right)$ has the $\Delta_{\perp} \longrightarrow-\Delta_{\perp}$ symmetry. $\Omega$ is the gauge link needed for color gauge invariance. In this work, we use the light-front gauge and take the gauge link to be unity. The symbol $\Gamma$ represents the Dirac matrix defining the types of quark densities.

In this work, we calculate the above Wigner distributions for a quark state dressed with a gluon. The state of momentum $p$ and helicity $\sigma$, can be expanded in Fock space in terms of multi-parton light-front wave functions (LFWFs) 20]

$$
\begin{array}{r}
\left|p^{+}, p_{\perp}, \sigma\right\rangle=\Phi^{\sigma}(p) b_{\sigma}^{\dagger}(p)|0\rangle+\sum_{\sigma_{1} \sigma_{2}} \int\left[d p_{1}\right] \int\left[d p_{2}\right] \sqrt{16 \pi^{3} p^{+}} \delta^{3}\left(p-p_{1}-p_{2}\right) \\
\Phi_{\sigma_{1} \sigma_{2}}^{\sigma}\left(p ; p_{1}, p_{2}\right) b_{\sigma_{1}}^{\dagger}\left(p_{1}\right) a_{\sigma_{2}}^{\dagger}\left(p_{2}\right)|0\rangle ;
\end{array}
$$

where $[d p]=\frac{d p^{+} d^{2} p_{\perp}}{\sqrt{16 \pi^{3} p^{+}}} . \Phi^{\sigma}(p)$ and $\Phi_{\sigma_{1} \sigma_{2}}^{\sigma}$ are the single particle (quark) and two particle (quarkgluon) light-front wave function (LFWF). $\sigma_{1}$ and $\sigma_{2}$ are the helicities of the quark and gluon respectively. $\Phi^{\sigma}(p)$ gives the wave function renormalization for the quark. The two particle function $\Phi_{\sigma_{1} \sigma_{2}}^{\sigma}\left(p ; p_{1}, p_{2}\right)$ gives the probability to find a bare quark having momentum $p_{1}$ and helicity $\sigma_{1}$ and a bare gluon with momentum $p_{2}$ and helicity $\sigma_{2}$ in the dressed quark. The two particle LFWF is related to the boost invariant LFWF; $\Psi_{\sigma_{1} \sigma_{2}}^{\sigma}\left(x, q_{\perp}\right)=\Phi_{\sigma_{1} \sigma_{2}}^{\sigma} \sqrt{P^{+}}$. Here we have used the Jacobi momenta $\left(x_{i}, q_{i \perp}\right)$ :

$$
p_{i}^{+}=x_{i} p^{+}, \quad q_{i \perp}=k_{i \perp}+x_{i} p_{\perp}
$$


so that $\sum_{i} x_{i}=1, \sum_{i} q_{i \perp}=0$. These two-particle LFWFs be calculated perturbatively as [20]:

$$
\begin{array}{r}
\Psi_{\sigma_{1} \sigma_{2}}^{\sigma a}\left(x, q_{\perp}\right)=\frac{1}{\left[m^{2}-\frac{m^{2}+\left(q_{\perp}\right)^{2}}{x}-\frac{\left(q_{\perp}\right)^{2}}{1-x}\right]} \frac{g}{\sqrt{2(2 \pi)^{3}}} T^{a} \chi_{\sigma_{1}}^{\dagger} \frac{1}{\sqrt{1-x}} \\
{\left[-2 \frac{q_{\perp}}{1-x}-\frac{\left(\sigma_{\perp} \cdot q_{\perp}\right) \sigma_{\perp}}{x}+\frac{i m \sigma_{\perp}(1-x)}{x}\right] \chi_{\sigma}\left(\epsilon_{\perp \sigma_{2}}\right)^{*} .}
\end{array}
$$

We use the two component formalism [16]; $\chi$ is the two component spinor, $T^{a}$ are the color $S U(3)$ matrices, $m$ is the mass of the quark and $\epsilon_{\perp \sigma_{2}}$ is the polarization vector of the gluon; $\perp=1,2$. As stated in the introduction, the quark state dressed by a gluon as we consider here mimics the bound state of a spin-1/2 particle and a spin-1 particle. For such a bound state the bound state mass $M$ should be less than the sum of the masses of the constituents for stability. Here in the two-component formalism, we use the same mass for the bare as well as the dressed quark in perturbation theory [17]. We investigate the Wigner distributions for unpolarized and longitudinally polarized dressed quark and the relevant correlators are with $\Gamma=\gamma^{+}$and $\gamma^{+} \gamma_{5}$. The single particle sector contributes through the normalization of the state, which is important to get the complete contribution at $x=1$. In this work we restrict ourselves to the kinematic region $x<1$, and in this case the contribution from $\Phi^{\sigma}(p)$ can be taken to be 1 . We calculate the contribution to the quark-quark correlator and the Wigner distribution from the two particle sector in the Fock space expansion. This is given by

$$
\begin{gathered}
W^{\left[\gamma^{+}\right]}\left(\Delta_{\perp}, k_{\perp}, x, \sigma\right)=\frac{1}{(2 \pi)^{3}} \sum_{\sigma_{1}, \sigma_{2}} \Psi_{\sigma_{1} \sigma_{2}}^{* \sigma a}\left(x, q_{\perp}^{\prime}\right) \Psi_{\sigma_{1} \sigma_{2}}^{\sigma a}\left(x, q_{\perp}\right), \\
W^{\left[\gamma^{+} \gamma_{5}\right]}\left(\Delta_{\perp}, k_{\perp}, x, \sigma\right)=\frac{1}{(2 \pi)^{3}} \sum_{\sigma_{1}, \sigma_{2}, \lambda_{1}} \Psi_{\lambda_{1} \sigma_{2}}^{* \sigma a}\left(x, q_{\perp}^{\prime}\right) \chi_{\lambda_{1}}^{\dagger} \sigma_{3} \chi_{\sigma_{1}} \Psi_{\sigma_{1} \sigma_{2}}^{\sigma a}\left(x, q_{\perp}\right) ;
\end{gathered}
$$

where the Jacobi relation for the transverse momenta in the symmetric frame is given by $q_{\perp}^{\prime}=k_{\perp}-\frac{\Delta_{\perp}}{2}(1-x)$ and $q_{\perp}=k_{\perp}+\frac{\Delta_{\perp}}{2}(1-x)$. We use the symbol $\rho_{\lambda \lambda^{\prime}}$ for Wigner distributions, where $\lambda\left(\lambda^{\prime}\right)$ is longitudinal polarization of target state(quark). The four Wigner distributions have been defined in [7] as

$$
\rho_{U U}\left(b_{\perp}, k_{\perp}, x\right)=\frac{1}{2}\left[\rho^{\left[\gamma^{+}\right]}\left(b_{\perp}, k_{\perp}, x,+e_{z}\right)+\rho^{\left[\gamma^{+}\right]}\left(b_{\perp}, k_{\perp}, x,-e_{z}\right)\right]
$$


is the Wigner distribution of unpolarized quarks in unpolarized target state.

$$
\rho_{L U}\left(b_{\perp}, k_{\perp}, x\right)=\frac{1}{2}\left[\rho^{\left[\gamma^{+}\right]}\left(b_{\perp}, k_{\perp}, x,+e_{z}\right)-\rho^{\left[\gamma^{+}\right]}\left(b_{\perp}, k_{\perp}, x,-e_{z}\right)\right]
$$

is the distortion due to longitudinal polarization of the target state.

$$
\rho_{U L}\left(b_{\perp}, k_{\perp}, x\right)=\frac{1}{2}\left[\rho^{\left[\gamma^{+} \gamma_{5}\right]}\left(b_{\perp}, k_{\perp}, x,+e_{z}\right)+\rho^{\left[\gamma^{+} \gamma_{5}\right]}\left(b_{\perp}, k_{\perp}, x,-e_{z}\right)\right]
$$

represents distortion due to the longitudinal polarization of quarks, and

$$
\rho_{L L}\left(b_{\perp}, k_{\perp}, x\right)=\frac{1}{2}\left[\rho^{\left[\gamma^{+} \gamma_{5}\right]}\left(b_{\perp}, k_{\perp}, x,+e_{z}\right)-\rho^{\left[\gamma^{+} \gamma_{5}\right]}\left(b_{\perp}, k_{\perp}, x,-e_{z}\right)\right]
$$

represents the distortion due to the correlation between the longitudinal polarized target state and quarks.

In our case, $+e_{z}$ and $-e_{z}$ correspond to helicity up and down of the target state, respectively. In the model we consider, $\rho_{L U}=\rho_{U L}$ and the final expression for the three independent Wigner distribution are as follows:

$$
\begin{gathered}
\rho_{U U}^{\left[\gamma^{+}\right]}\left(b_{\perp}, k_{\perp}, x\right)=N \int d \Delta_{x} \int d \Delta_{y} \frac{\cos \left(\Delta_{\perp} \cdot b_{\perp}\right)}{D\left(q_{\perp}\right) D\left(q_{\perp}^{\prime}\right)}\left[I_{1}+\frac{4 m^{2}(1-x)}{x^{2}}\right] \\
\rho_{L U}^{\left[\gamma^{+}\right]}\left(b_{\perp}, k_{\perp}, x\right)=N \int d \Delta_{x} \int d \Delta_{y} \frac{\sin \left(\Delta_{\perp} \cdot b_{\perp}\right)}{D\left(q_{\perp}\right) D\left(q_{\perp}^{\prime}\right)}\left[4\left(k_{x} \Delta_{y}-k_{y} \Delta_{x}\right) \frac{(1+x)}{x^{2}(1-x)}\right] \\
\rho_{L L}^{\left[\gamma^{+} \gamma_{5}\right]}\left(b_{\perp}, k_{\perp}, x\right)=N \int d \Delta_{x} \int d \Delta_{y} \frac{\cos \left(\Delta_{\perp} \cdot b_{\perp}\right)}{D\left(q_{\perp}\right) D\left(q_{\perp}^{\prime}\right)}\left[I_{1}-\frac{4 m^{2}(1-x)}{x^{2}}\right]
\end{gathered}
$$

where $A_{x}, A_{y}$ are $x, y$ component of $A_{\perp}$ and

$$
D\left(k_{\perp}\right)=\left(m^{2}-\frac{m^{2}+\left(k_{\perp}\right)^{2}}{x}-\frac{\left(k_{\perp}\right)^{2}}{1-x}\right) \quad I_{1}=4\left(\left(k_{\perp}\right)^{2}-\frac{\Delta_{\perp}^{2}(1-x)^{2}}{4}\right) \frac{\left(1+x^{2}\right)}{x^{2}(1-x)^{3}} .
$$

Wigner distribution are real [7, which is due to the Hermiticity property of the GTMDs to which they are related; and in the above expressions, we have taken the real part of the Fourier transforms. 


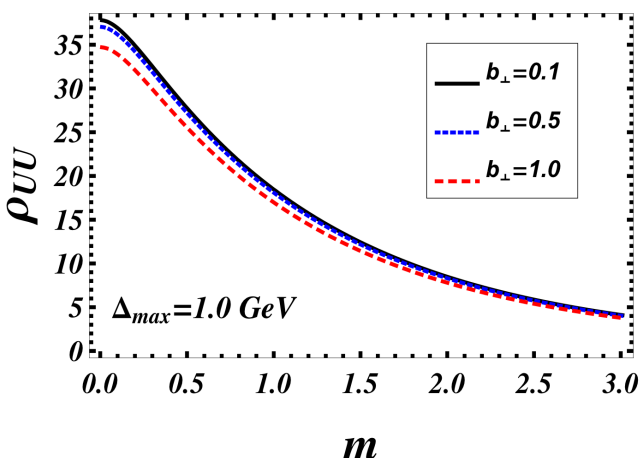

(a)

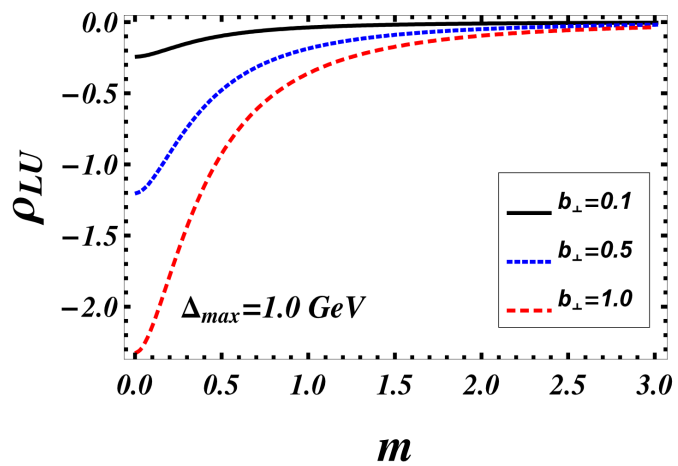

(c)

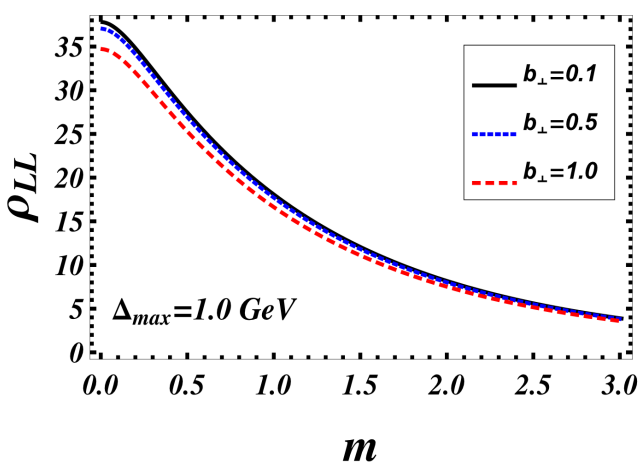

(e)

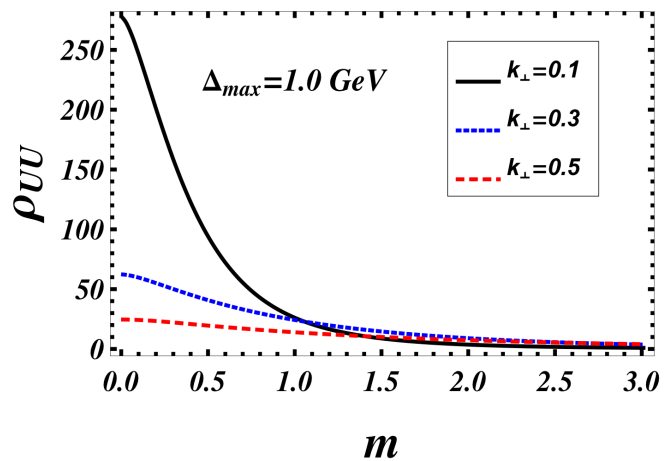

(b)

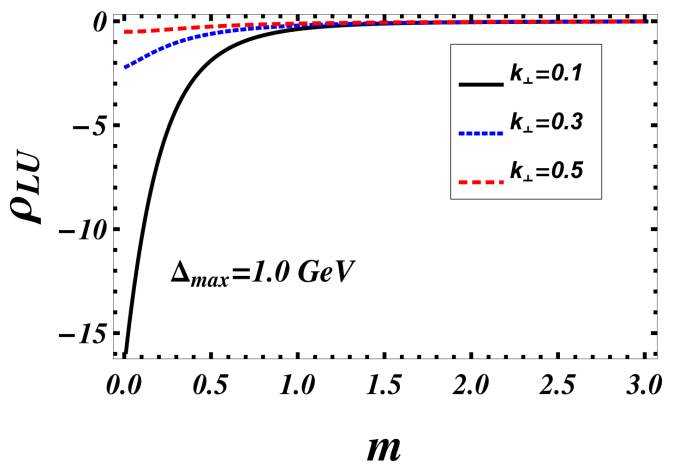

(d)

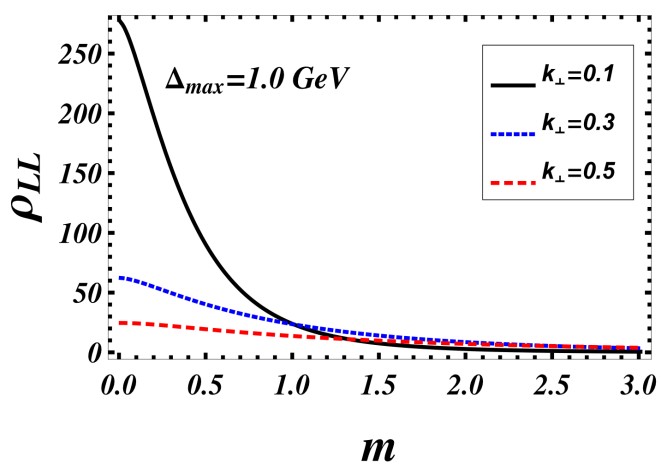

FIG. 1: (Color online) Plots of the Wigner distributions vs m (mass in $G e V$ ) for fixed values of $b_{\perp}$ and $k_{\perp}$ at $\Delta_{\max }=1.0 \mathrm{GeV}$. All the plots on the left $(\mathrm{a}, \mathrm{c}, \mathrm{e})$ are for three fixed values of $b_{\perp}(0.1,0.5,1.0)$ in $G e V^{-1}$ where $k_{\perp}=0.4 \mathrm{GeV}$. Plots on the right $(\mathrm{b}, \mathrm{d}, \mathrm{f})$ are for three fixed values of $k_{\perp}(0.1,0.3,0.5)$ in $G e V$ where and $b_{\perp}=0.4 G e V^{-1}$. For all plots we took $\overrightarrow{k_{\perp}}=k \hat{j}$ and $\overrightarrow{b_{\perp}}=b \hat{j}$.

\section{ORBITAL ANGULAR MOMENTUM OF QUARKS}

In [4] it has been shown that the quark-quark correlator in Eq. (2) defining the Wigner distributions can be parameterized in terms of generalized transverse momentum dependent parton 

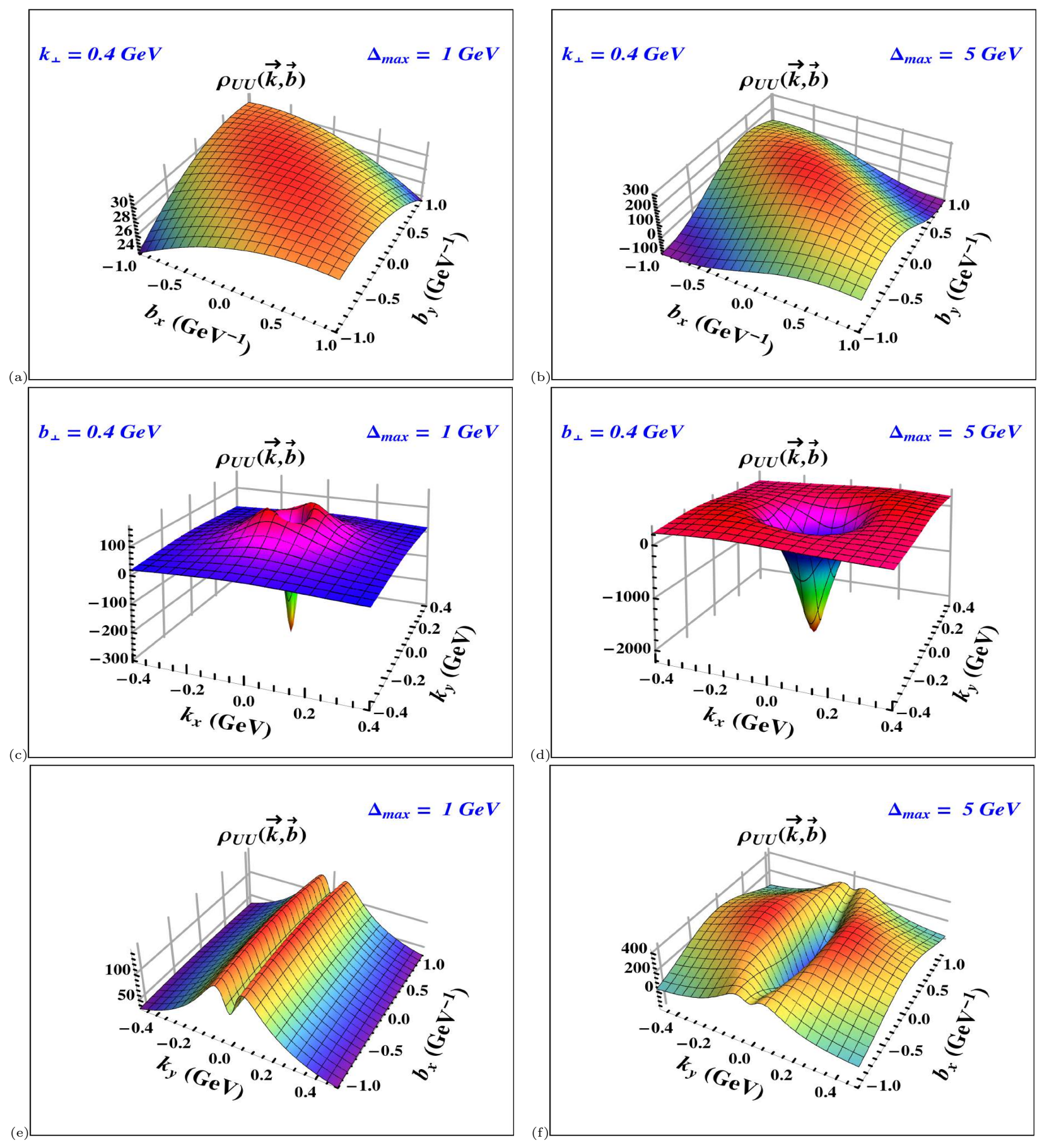

FIG. 2: (Color online) 3D plots of the Wigner distributions $\rho_{U U}$. Plots (a) and (b) are in $b$ space with $k_{\perp}=0.4 \mathrm{GeV}$. Plots (c) and (d) are in $k$ space with $b_{\perp}=0.4 \mathrm{GeV}^{-1}$. Plots (e) and (f) are in mixed space where $k_{x}$ and $b_{y}$ are integrated. All the plots on the left panel (a,c,e) are for $\Delta_{\max }=1.0 \mathrm{GeV}$. Plots on the right panel $(\mathrm{b}, \mathrm{d}, \mathrm{f})$ are for $\Delta_{\max }=5.0 \mathrm{GeV}$. For all the plots we kept $m=0.33 \mathrm{GeV}$, integrated out the $x$ variable and we took $\overrightarrow{k_{\perp}}=k \hat{j}$ and $\overrightarrow{b_{\perp}}=b \hat{j}$. 

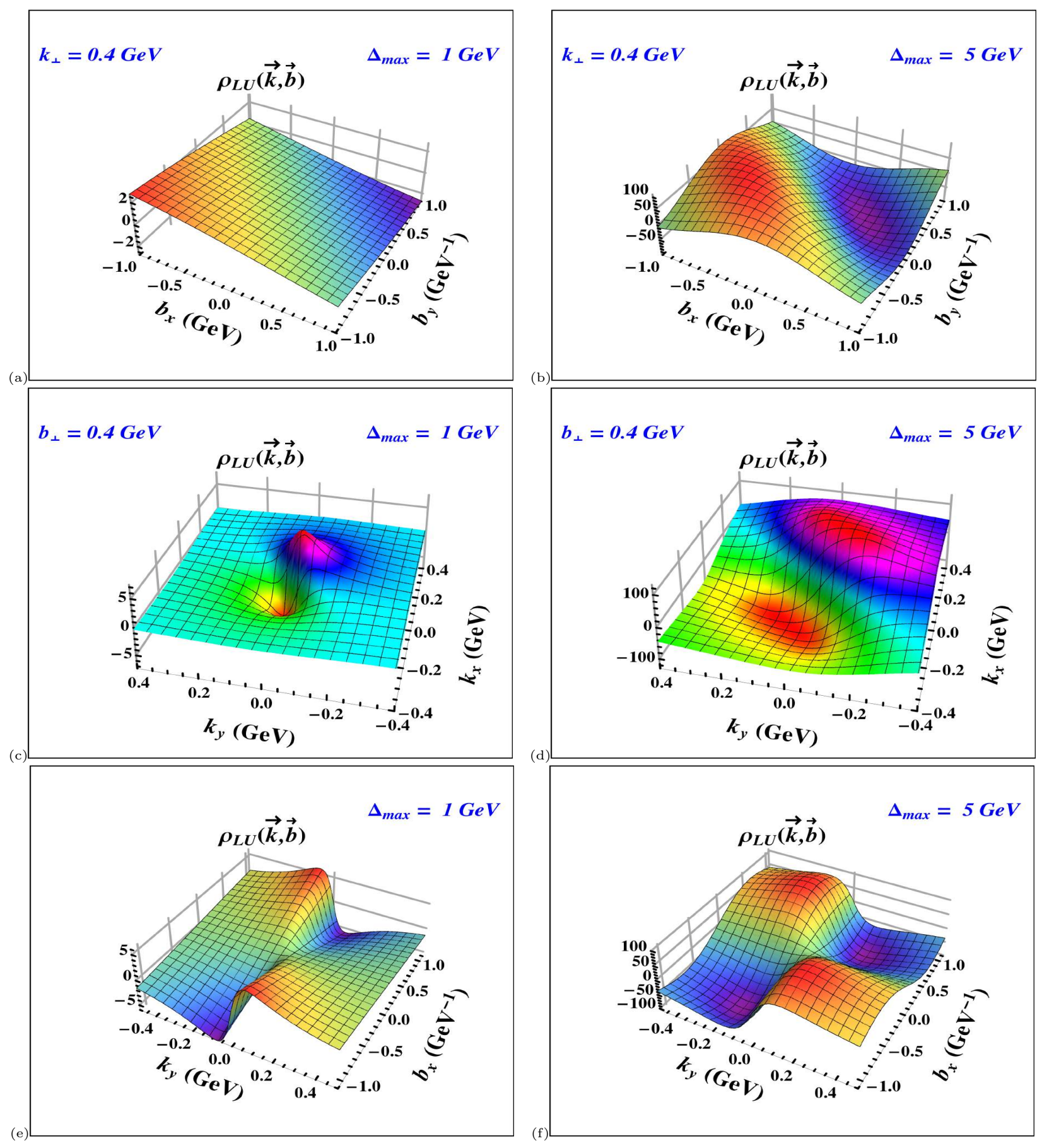

FIG. 3: (Color online) 3D plots of the Wigner distributions $\rho_{L U}$. Plots (a) and (b) are in $b$ space with $k_{\perp}=0.4 \mathrm{GeV}$. Plots (c) and (d) are in $k$ space with $b_{\perp}=0.4 \mathrm{GeV}^{-1}$. Plots (e) and (f) are in mixed space where $k_{x}$ and $b_{y}$ are integrated. All the plots on the left panel (a,c,e) are for $\Delta_{\max }=1.0 \mathrm{GeV}$. Plots on the right panel $(\mathrm{b}, \mathrm{d}, \mathrm{f})$ are for $\Delta_{\max }=5.0 \mathrm{GeV}$. For all the plots we kept $m=0.33 \mathrm{GeV}$, integrated out the $x$ variable and we took $\overrightarrow{k_{\perp}}=k \hat{j}$ and $\overrightarrow{b_{\perp}}=b \hat{j}$. 

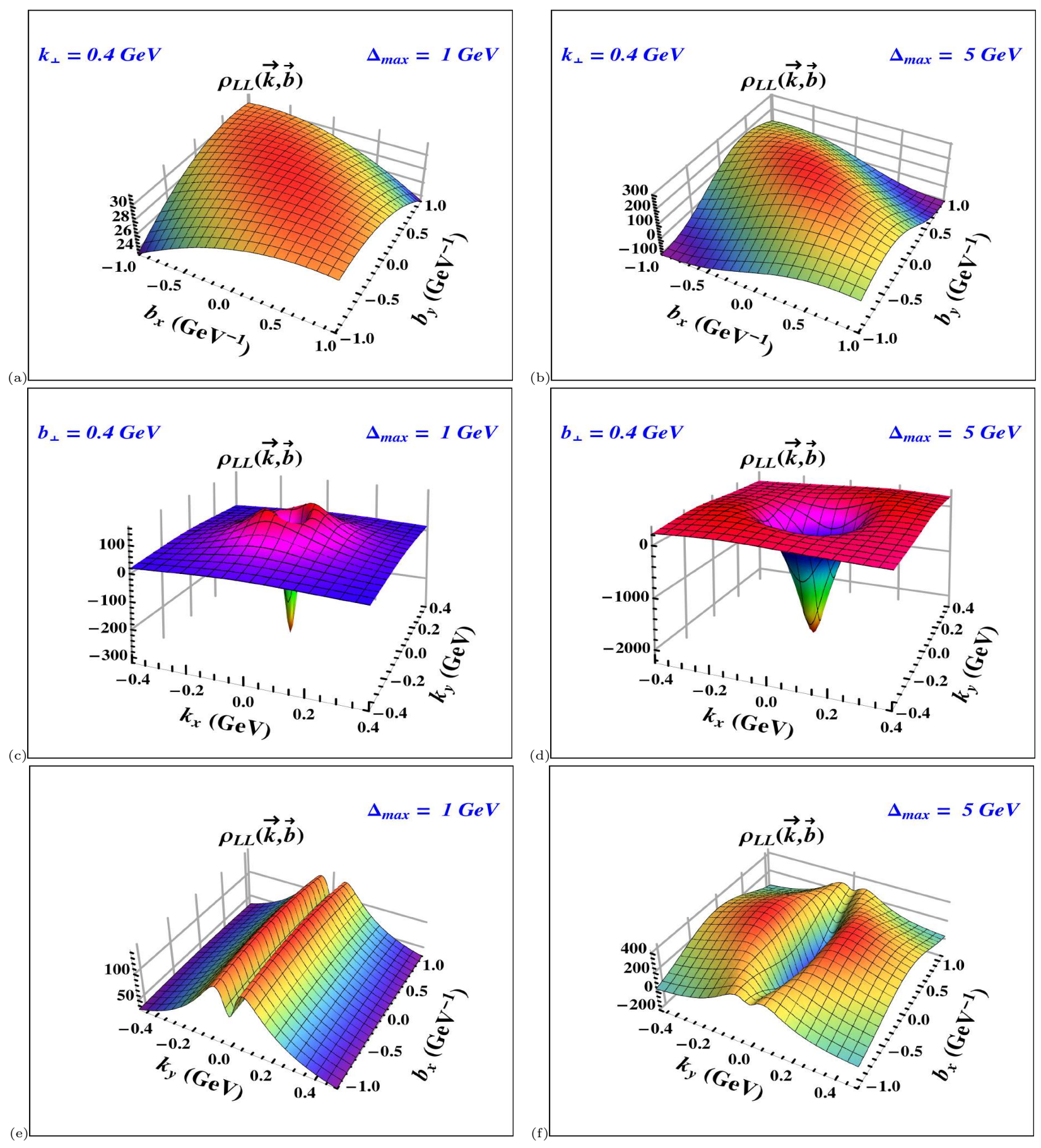

FIG. 4: (Color online) 3D plots of the Wigner distributions $\rho_{L L}$. Plots (a) and (b) are in $b$ space with $k_{\perp}=0.4 \mathrm{GeV}$. Plots (c) and (d) are in $k$ space with $b_{\perp}=0.4 \mathrm{GeV}^{-1}$. Plots (e) and (f) are in mixed space where $k_{x}$ and $b_{y}$ are integrated. All the plots on the left panel $(\mathrm{a}, \mathrm{c}, \mathrm{e})$ are for $\Delta_{\max }=1.0 \mathrm{GeV}$. Plots on the right panel $(\mathrm{b}, \mathrm{d}, \mathrm{f})$ are for $\Delta_{\max }=5.0 \mathrm{GeV}$. For all the plots we kept $m=0.33 \mathrm{GeV}$, integrated out the $x$ variable and we took $\overrightarrow{k_{\perp}}=k \hat{j}$ and $\overrightarrow{b_{\perp}}=b \hat{j}$. 
distributions (GTMDs). For the twist two case we have four GTMDs $\left(F_{1, i}\right)$ corresponding to $\gamma^{+}$and four more for $\gamma^{+} \gamma_{5}\left(G_{1, i}\right)$

$$
W_{\lambda, \lambda^{\prime}}^{\left[\gamma^{+}\right]}=\frac{1}{2 M} \bar{u}\left(p^{\prime}, \lambda^{\prime}\right)\left[F_{1,1}-\frac{i \sigma^{i+} k_{i \perp}}{P^{+}} F_{1,2}-\frac{i \sigma^{i+} \Delta_{i \perp}}{P^{+}} F_{1,3}+\frac{i \sigma^{i j} k_{i \perp} \Delta_{j \perp}}{M^{2}} F_{1,4}\right] u(p, \lambda)
$$

$W_{\lambda, \lambda^{\prime}}^{\left[\gamma^{+} \gamma_{5}\right]}=\frac{\bar{u}\left(p^{\prime}, \lambda^{\prime}\right)}{2 M}\left[\frac{-i \epsilon_{\perp}^{i j} k_{i \perp} \Delta_{j \perp}}{M^{2}} G_{1,1}-\frac{i \sigma^{i+} \gamma_{5} k_{i \perp}}{P^{+}} G_{1,2}-\frac{i \sigma^{i+} \gamma_{5} \Delta_{i \perp}}{P^{+}} G_{1,3}+i \sigma^{+-} \gamma_{5} G_{1,4}\right] u(p, \lambda)$.

Using the above two equations and Eq.(1) we calculate the GTMDs for the dressed quark model at twist two. We have used the Bjorken and Drell convention for gamma matrices. Using the two-particle LFWFs we obtain the final expression for the GTMDS as follows :

$$
\begin{aligned}
& F_{11}=-\frac{N\left[4 k_{\perp}^{2}\left(1+x^{2}\right)+(x-1)^{2}\left(4 m^{2}(x-1)^{2}-\left(1+x^{2}\right) \Delta_{\perp}^{2}\right)\right]}{D\left(q_{\perp}\right) D\left(q_{\perp}^{\prime}\right) 2 x^{2}(x-1)^{3}} ; \\
& F_{12}=\frac{2 N m^{2} \Delta_{\perp}^{2}}{D\left(q_{\perp}\right) D\left(q_{\perp}^{\prime}\right) x\left(k_{y} \Delta_{x}-k_{x} \Delta_{y}\right)} ; \\
& F_{13}=\frac{N}{D\left(q_{\perp}\right) D\left(q_{\perp}^{\prime}\right) 4 x\left(k_{y} \Delta_{x}-k_{x} \Delta_{y}\right)} \\
& \quad\left[8 m^{2}\left(k_{\perp} \Delta_{\perp}\right)-\frac{\left(k_{y} \Delta_{x}-k_{x} \Delta_{y}\right)\left(4 k_{\perp}^{2}\left(1+x^{2}\right)+(x-1)^{2}\left(4 m^{2}(x-1)^{2}-\left(1+x^{2}\right) \Delta_{\perp}^{2}\right)\right)}{x(x-1)^{3}}\right] \\
& F_{14}=\frac{2 N m^{2}(1+x)}{D\left(q_{\perp}\right) D\left(q_{\perp}^{\prime}\right) x^{2}(1-x)} .
\end{aligned}
$$




$$
\begin{aligned}
& G_{11}=-\frac{2 N m^{2}(1+x)}{D\left(q_{\perp}\right) D\left(q_{\perp}^{\prime}\right) x^{2}(x-1)} ; \\
& G_{12}=\frac{-N}{D\left(q_{\perp}\right) D\left(q_{\perp}^{\prime}\right) x(x-1)}\left[4 m^{2} \frac{k_{\perp} \cdot \Delta_{\perp}}{\left(k_{y} \Delta_{x}-k_{x} \Delta_{y}\right)}-\frac{(1+x) \Delta_{\perp}^{2}}{x}\right] ; \\
& G_{13}=\frac{N\left[(1+x)\left(\Delta_{y}^{2}-\Delta_{x}^{2}+\Delta_{x} \Delta_{y}\left(k_{y}^{2}-k_{x}^{2}\right)\right)+4 x m^{2} k_{\perp}^{2}\right]}{D\left(q_{\perp}\right) D\left(q_{\perp}^{\prime}\right) x^{2}(x-1)\left(k_{y} \Delta_{x}-k_{x} \Delta_{y}\right)} ; \\
& G_{14}=\frac{N\left[-4 k_{\perp}^{2}\left(1+x^{2}\right)+(x-1)^{2}\left(4 m^{2}(x-1)^{2}-\left(1+x^{2}\right) \Delta_{\perp}^{2}\right)\right]}{D\left(q_{\perp}\right) D\left(q_{\perp}^{\prime}\right) 2 x^{2}(x-1)^{3}} ;
\end{aligned}
$$

where $N=\frac{g^{2} C_{f}}{2(2 \pi)^{3}}$ is the normalization constant and $C_{f}$ is the color factor.

The kinetic quark orbital angular momentum (OAM) is given in terms of the GPDs [21] as:

$$
L_{z}^{q}=\frac{1}{2} \int d x\left\{x\left[H^{q}(x, 0,0)+E^{q}(x, 0,0)\right]-\tilde{H}^{q}(x, 0,0)\right\} .
$$

The GPDs in the above equation are defined at $\xi=0$ or when the momentum transfer is purely in the transverse direction. GPDs in the model we consider have been already calculated in $[22,[23],[24,[25],[26]$. The kinetic OAM is related to the GTMDs [4] by the following relations:

$$
\begin{aligned}
& H(x, 0, t)=\int d^{2} k_{\perp} F_{11} ; \\
& E(x, 0, t)=\int d^{2} k_{\perp}\left[-F_{11}+2\left(\frac{k_{\perp} \cdot \Delta_{\perp}}{\Delta_{\perp}^{2}} F_{12}+F_{13}\right)\right] ; \\
& \tilde{H}(x, 0, t)=\int d^{2} k_{\perp} G_{14} .
\end{aligned}
$$



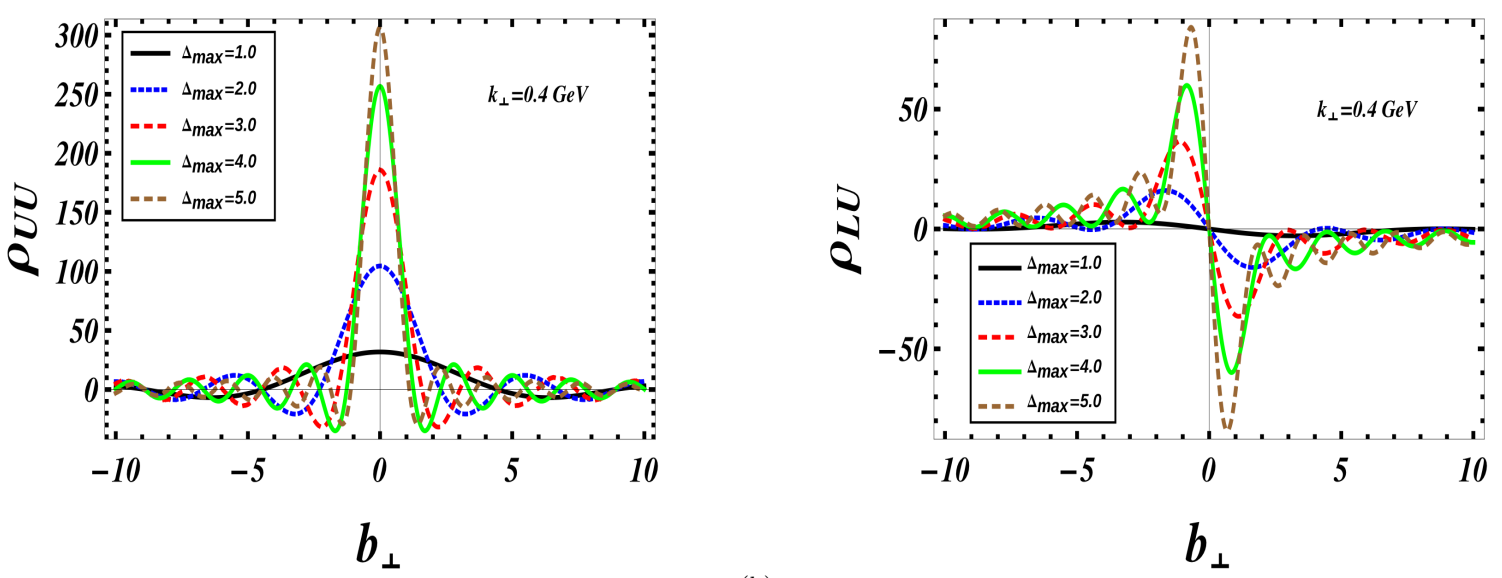

(a)

(b)

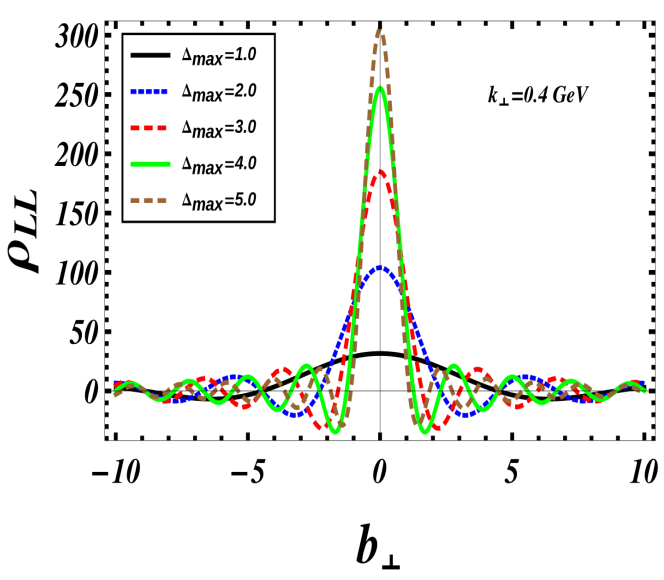

(c)

FIG. 5: (Color online) Plots of the Wigner distributions vs $b_{\perp}$ for different $\Delta_{\max }(G e V)$ for a fixed value $k_{\perp}=0.4 \mathrm{GeV}$ and $\mathrm{m}=0.33 \mathrm{GeV} . b_{\perp}$ is in $\mathrm{GeV}^{-1}$.

Using the GTMDs calculated we have the following final expression for the kinetic orbital angular momentum of quarks in the dressed quark model:

$$
L_{z}^{q}=\frac{N}{2} \int d x\left\{-f(x) I_{1}+4 m^{2}(1-x)^{2} I_{2}\right\}
$$

where, 


$$
\begin{aligned}
I_{1} & =\int \frac{d^{2} k_{\perp}}{m^{2}(1-x)^{2}+\left(k_{\perp}\right)^{2}}=\pi \log \left[\frac{Q^{2}+m^{2}(1-x)^{2}}{\mu^{2}+m^{2}(1-x)^{2}}\right] \\
I_{2} & =\int \frac{d^{2} k_{\perp}}{\left(m^{2}(1-x)^{2}+\left(k_{\perp}\right)^{2}\right)^{2}}=\frac{\pi}{\left(m^{2}(1-x)^{2}\right)} \\
f(x) & =2\left(1+x^{2}\right) .
\end{aligned}
$$

Here $Q$ and $\mu$ are the upper and lower limits of the $k_{\perp}$ integration respectively. $Q$ is the large scale involved in the process, which comes from the large momentum cutoff in this approach [20]. Alternatively one can choose an invariant mass cutoff [18]. $\mu$ can be safely taken to be zero provided the quark mass is non-zero. In fact, we have taken $\mu$ to be zero.

The GTMDs $F_{14}$ and $G_{11}$ are not reducible to any GPDs or transverse-momentum dependent parton distributions (TMDs) in any limit. These appear purely at the level of the GTMDs and provide new information not contained in the GPDs or TMDs. $F_{14}$ is related to the canonical OAM as shown in [7, 12, 27]:

$$
l_{z}^{q}=-\int d x d^{2} k_{\perp} \frac{k_{\perp}^{2}}{m^{2}} F_{14} .
$$

We give the final expression for the canonical quark OAM in the dressed quark model.

$$
l_{z}^{q}=-2 N \int d x\left(1-x^{2}\right)\left[I_{1}-m^{2}(x-1)^{2} I_{2}\right]
$$

The above expression is in agreement with [20, where the authors have calculated the quark canonical OAM using the same model neglecting the quark mass. Our results are also in agreement with [28] as well as a recent calculation in [29]. We thus confirm the conclusion in [29] in our model calculation that the GTMDs $F_{14}$ and $G_{11}$ exist and non-zero, in contrast to the arguments given in [30]. Also in [29] the above two GTMDs were calculated incorporating the gauge link; as their results agree with ours, it is clear that the gauge link does not contribute to these GTMDs and the result is independent of the choice of the gauge link, which was also noted in [29]. 
As shown in [7, 31], the correlation between the quark spin and its OAM is given by

$$
C_{z}^{q}=\int d x d^{2} k_{\perp} \frac{k_{\perp}^{2}}{m^{2}} G_{11} .
$$

As in our model $F_{14}=-G_{11}$, the above correlation is given by Eq. (29). The spin-orbit correlation for the quark in the dressed quark is negative. This is opposite to what is observed in chiral quark-soliton model and constituent quark model, namely here the quark spin is anti-aligned with its OAM, unlike the other two models where there is no gluon.

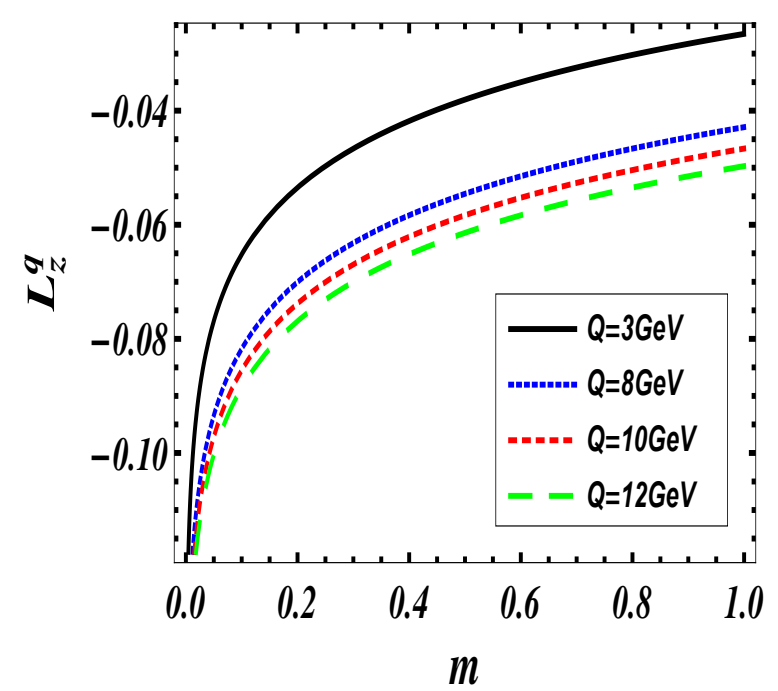

(a)

FIG. 6: (Color online) Plots of OAM (a) $L_{z}^{q}$ and (b) $l_{z}^{q}$ vs $m(\mathrm{GeV})$ for different values of $Q(\mathrm{GeV})$.

\section{NUMERICAL RESULTS}

In all plots, we have integrated over $x$ and divided by the normalization, $N$. In Fig. 1 we show the dependence of the Wigner distributions on the quark mass. We took the mass of the dressed quark to be the same as the bare quark. Here we have plotted the Wigner distributions versus the mass for fixed values of $b_{\perp}$ in $\mathrm{GeV}^{-1}$ and $k_{\perp}$ in $\mathrm{GeV}$. Ideally the upper limit of the $\Delta_{\perp}$ integration should be infinity. However we have imposed an upper cutoff $\Delta_{\max }$ in the numerical integration. In Fig. 1 we have taken $\Delta_{\max }=1.0 \mathrm{GeV}$. Here $\overrightarrow{b_{\perp}}=b \hat{j}$ and $\overrightarrow{k_{\perp}}=k \hat{j}$. For $\rho_{U U}$ in Fig. 1 (a) we have plotted the mass dependence for three different values of $b_{\perp}$ 
which are $0.1,0.5$ and $1.0 \mathrm{GeV}^{-1}$ keeping $k_{\perp}=0.4 \mathrm{GeV}$ and we see that the value decreases with increasing mass. This is because the mass term in the denominator of Eq. 12 coming from the $D(k)$ function is dominant over the other term. For larger $b_{\perp}$ values the distribution has smaller values as seen from the plot. In Fig. 1 (b) we have plotted the mass dependence for three different values of $k_{\perp}$ which are 0.1, 0.3 and $0.5 \mathrm{GeV}$ keeping $b_{\perp}=0.4 \mathrm{GeV}^{-1}$. Again we see the same behavior as in Fig. 1 (a), in the lower mass range $\rho_{U U}$ increases sharply for smaller $k_{\perp}$. In fig. 1 (c) and fig. 1 (d) we have plotted the mass dependence for $\rho_{L U}$ with the same settings as for $\rho_{U U}$. Since we choose $\overrightarrow{k_{\perp}}=k \hat{j}$ and because of the factor $k_{x} \Delta_{y}-k_{y} \Delta_{x}$ we observe that the distribution has negative values but we do observe the same behavior as seen previously. Lastly in Fig. 1 (e) and Fig. 1 (f) we show the results for $\rho_{L L}$. Since $\rho_{U U}$ and $\rho_{L L}$ only differ by a sign in their mass term as seen in Eqs.12, 14, the results are nearly identical, as the mass term gives sub-dominant contribution. In all the plots of 1 we observe that at higher mass range the distributions are nearly independent of $b_{\perp}$ and $k_{\perp}$ values.

In Fig. 2 we show the 3D plots for the Wigner distribution $\rho_{U U}$. In the numerical calculation for Eq 12 we have upper cut-off's $\Delta_{x}^{\max }$ and $\Delta_{y}^{\max }$ for the $\Delta_{\perp}$ integration. In all plots we have taken $m=0.33 \mathrm{GeV}$. In Figs. 2 (a) and (b) we have plotted $\rho_{U U}$ in $b$ space with $k_{\perp}=0.4 \mathrm{GeV}$ such that $\overrightarrow{k_{\perp}}=k \hat{j}$ for $\Delta_{\perp}^{\max }=1.0 \mathrm{GeV}$ and $\Delta_{\perp}^{\max }=5.0 \mathrm{GeV}$ respectively. We see that the plot has a peak centered at $b_{x}=b_{y}=0$ decreasing in the outer regions of the $b$ space. In [7] the authors have shown that the contour plots show asymmetry associated with the orbital angular momentum and the asymmetry favored the $b \perp k$ direction to $b \| k$. This can be understood from semi-classical arguments in a model with confinement. As no confining potential is present in the perturbative model we consider here, the behavior is expected to be different. In our case we observe the asymmetry but there is no particular favored direction for this asymmetry. In Figs. 2 (c) and (d) we have plots in the $k$ space where $b_{\perp}=0.4 \mathrm{GeV}$ such that $\overrightarrow{b_{\perp}}=b \hat{j}$ for $\Delta_{\perp}^{\max }=1.0 \mathrm{GeV}$ and $\Delta_{\perp}^{\max }=5.0 \mathrm{GeV}$ respectively. The behavior in the $k$ space is similar to that in the $b$ space but the peaks have negative values. In Fig. 2 (e) and (f) we show the plots in the mixed space. As discussed earlier, Wigner distributions do not have probability interpretation due to uncertainty principle in quantum mechanics. However in the distributions $\rho_{U U}\left(k_{y}, b_{x}\right)$ we have integrated out the $k_{x}$ and $b_{y}$ dependence giving us the probability densities correlating $k_{y}$ and $b_{x}$, this correlation is not restricted by uncertainty principle. Unlike in [7] 
we observe a minima at $b_{x}=0$ and $k_{y}=0$. In fact the minima is observed for all $b_{x}$ values for $k_{y}=0$. As $\Delta_{\max }$ increases the minima gets deeper. The plots show that the probability of finding a quark with fixed $k_{y}$ and $b_{x}$ first increases away from $k_{y}=0$ and then decreases.

In Fig. 3 we show the 3D plots for the Wigner distribution $\rho_{L U}$. This is the distortion of the Wigner distribution of unpolarized quarks due to the longitudinal polarization of the dressed quark. In fig. 3 (a) and (b) we have plotted $\rho_{L U}$ in $b$ space with $k_{\perp}=0.4 G e V$ such that $\overrightarrow{k_{\perp}}=k \hat{j}$ for $\Delta_{\perp}^{\max }=1.0 \mathrm{GeV}$ and $\Delta_{\perp}^{\max }=5.0 \mathrm{GeV}$ respectively. Like in [7] we observe a dipole structure in these plots and the dipole magnitude increases with increase in $\Delta_{\max }$. In Fig. 3 (c) and (d) we have plots in the $k$ space where $b_{\perp}=0.4 \mathrm{GeV}$ such that $\overrightarrow{b_{\perp}}=b \hat{j}$ for $\Delta_{\perp}^{\max }=1.0 \mathrm{GeV}$ and $\Delta_{\perp}^{\max }=5.0 \mathrm{GeV}$ respectively. Again we observe a dipole structure but the orientation is rotated in the $k$ space when compared to the $b$ space plots of Fig. 3 (a) and Fig. 3 (b). As before the dipole magnitude increases with increase in $\Delta_{\max }$. In Fig. 3 (e) and (f) we show the plots in the mixed space. We observe the quadrupole structure in the mixed space like in [7] and the peaks increase in magnitude with increasing $\Delta_{\perp}^{\max }$.

In Fig. 4 we show the 3D plots for the Wigner distribution $\rho_{L L}$. The behavior is similar to that of Fig 2 since the Wigner distribution functions $\rho_{U U}$ and $\rho_{L L}$ only differ by the sign of the mass term in the numerator.

In Fig.5 we have plotted the dependence of the Wigner distributions on the upper limit of $\Delta_{\perp}$ integration. Ideally, the upper limit of the FT should be infinite, but for practical purpose, a finite upper limit is necessary. For physical processes, for example in the deeply virtual Compton scattering (DVCS) such limits are there from the kinematics, that is the momentum transfer should be much less than the virtuality of the photon, Q. Figs. 5(a), (b) and (c) show plots of $\rho_{U U}, \rho_{L U}$ and $\rho_{L L}$ respectively as functions of $b_{\perp}$ for a fixed value of $k_{\perp}$ and different values of $\Delta_{\max } . \rho_{U U}$ and $\rho_{L L}$ show similar behavior, which is expected from the analytic formulas. Both of them show a peak at $\left|b_{\perp}\right|=0$, the peak becomes sharper as $\Delta_{\max }$ increases. $\rho_{L U}$ is zero at $b_{\perp}=0$ and changes sign at the origin. Here we observe two peaks, and these move closer to $\left|b_{\perp}\right|=0$ as $\Delta_{\max }$ increases. This means that the correlations 
between the unpolarized quarks inside the unpolarized target as well as the distortions due to the longitudinal polarization of the quarks in the longitudinally polarized dressed quark target are large in the close vicinity of $b_{\perp}=0$ for fixed $k_{\perp}$. If the allowed transverse momentum transfer is higher, these correlations move closer to the origin. The distortions of the Wigner functions due to the longitudinal polarization of the quark in an unpolarized target changes sign for negative $b_{\perp}$, these distortions are related to the OAM of the quark. Such distortions are also more concentrated near the origin in $b$ space as the transverse momentum transfer is higher. Similar conclusion can be drawn on the spin-orbit correlation of the quark.

In Fig $[$ we have shown the orbital angular momentum of quarks as a function of the mass. Fig. 6 (a) is for $L_{z}^{q}$ and 6 (b) for $l_{z}^{q}$. Both the plots are shown for different values of $Q$ in $\mathrm{GeV}$ where $Q$ is the upper limit in the transverse momentum integration. As stated above, this is the large momentum scale involved in the process. We see similar qualitative behavior of $L_{z}^{q}$ and $l_{z}^{q}$ where both are giving negative values for the chosen domain of mass and also both the OAM decreases in magnitude with increasing mass. However the magnitude of the two OAM differs in our model, unlike the case in [7], where the same had been calculated in several models without any gluonic degrees of freedom and the total quark contribution to the OAM were equal for both cases. It is to be noted that there is only one quark flavor in the simple model we consider. In [7, the contribution to the OAM from different quark flavors were found to be different, but the sum over all flavors were equal for the two definitions of OAM. Also, in [28] it has been shown that a simple model without the gauge field (for example a scalar diquark model) gives the same result for the above two definitions of quark OAM. Thus the perturbative model we consider here explicitly shows the contribution of the gluonic degrees of freedom to the OAM, which has been calculated in [20, 29]. In fact in [20] it was shown that in the model considered here, after the inclusion of the single particle sector of the Fock space (which contributes at $x=1$ ), the gluon intrinsic helicity contribution to the helicity sum rule cancels the contribution from the canonical quark and gluon OAM and the Jaffe-Manohar helicity sum rule is satisfied. 


\section{CONCLUSION}

In this work, we calculated the Wigner distributions for a quark state dressed with a gluon using the overlap representation in terms of the LFWFs. This is a simple composite spin$1 / 2$ system which has a gluonic degree of freedom. Although the Wigner distributions in quantum mechanics are not measurable and do not have probabilistic interpretation, after integrating out some of the variables a probabilistic interpretation is possible to obtain. We calculated the Wigner distributions both for unpolarized and longitudinally polarized target and quarks and showed the correlations in transverse momentum and position space. We compared and contrasted the results with an earlier calculation of Wigner distributions in light cone constituent quark model and light-cone chiral quark soliton model. We also calculated the

kinetic quark OAM using the GPD sum rule and the canonical OAM and showed that these are different in magnitude, the difference is an effect of the gluonic degree of freedom. We also found that in the limit of zero quark mass our result for the canonical OAM agrees with that of [20]. We also presented the results for the spin-orbit correlation of the quark. Further work would involve calculating the Wigner distributions for the gluons and also including transverse polarization of the target and the quark.

\section{ACKNOWLEDGMENTS}

We would like to thank C. Lorce and B. Pasquini for helpful discussion. This work is supported by the DST project SR/S2/HEP-029/2010, Govt. of India. After we had put this paper in the arXiv, we became aware of [29] which appeared in the arXiv a couple of days before.

[1] E.P. Wigner, Phys.Rev. 40, 749 (1932).

[2] X. Ji, Phys. Rev. Lett. 91, 062001 (2003).

[3] A. Belitsky, X. Ji, F. Yuan; Phys.Rev. D 69, 074014 (2004).

[4] S.Meissner, A.Metz,and M. Schlegel, JHEP 08 (2009) 056; S.Meissner, A.Metz, M. Schlegel and K. Goeke, JHEP 08 (2008) 038. 
[5] C. Lorce and B. Pasquini, JHEP 09, 138 (2013).

[6] C. Lorce, B. Pasquini and M. Vanderhaeghen, JHEP 05, 041 (2011).

[7] C. Lorce, B.Pasquini, Phys. Rev. D84, 014015 (2011).

[8] A. Harindranath, Lectures given at the International School on Light-front Quantization and Non-perturbative QCD, hep-ph/9612244.

[9] J. Ashman et al., Nucl. Phys. B328, 1 (1989).

[10] E. Leader, C. Lorce, arXiv:1309.4235 hep-ph]; and the references therein.

[11] M. Burkardt, Phys. Rev. D 88, 014014 (2013).

[12] Y. Hatta, Phys. Lett. B 708, 186 (2012).

[13] C. Lorce, Phys. Lett. B 719, 185 (2013).

[14] Y. Hatta and S. Yoshida, JHEP 1210, 080 (2012).

[15] X. Ji, X. Xiong, F. Yuan, Phys. Rev. D 88, 014041 (2013).

[16] W-M. Zhang and Harindranath, Phys. Rev. D48, 4881 (1993).

[17] A. Harindranath, R. Kundu, W-M. Zhang, Phys.Rev. D 59, 094012 (1999); 094013 (1999).

[18] S. J. Brodsky, D. S. Hwang, B-Q. Ma, I. Schmidt, Nucl. Phys. B 593, 311 (2001).

[19] S. J. Brodsky, M. Diehl, D. S. Hwang, Nucl. Phys. B596, 99, (2001).

[20] A.Harindranath and R.Kundu, Phys. Rev. D59, 116013 (1999).

[21] X. Ji, Phys. Rev. Lett. 78,610 (1997).

[22] D. Chakrabarti, A. Mukherjee, Phys. Rev. D 71, 014038 (2005).

[23] D. Chakrabarti, A. Mukherjee, Phys. Rev. D 72, 034013 (2005).

[24] S. J. Brodsky, D. Chakrabarti, A. Harindranath, A. Mukherjee, J. P. Vary, Phys. Lett. B 641, 440 (2006).

[25] S. J. Brodsky, D. Chakrabarti, A. Harindranath, A. Mukherjee, J. P. Vary, Phys. Rev. D 75, $014003(2007)$.

[26] S. Meissner, A. Metz, K. Goeke, Phys. Rev. D76, 034002 (2007).

[27] C. Lorce, B. Pasquini, X. Xiong, F. Yuan, Phys. Rev. D 85, 114006 (2012).

[28] Hikmat BC, M. Burkardt, Few Body Syst. 52, 389 (2012).

[29] K. Kanazawa, C. Lorce, A. Metz, B. Pasquini, M. Schlegel, arXiv: 1403.5226[hep-ph].

[30] A. Courtoy, G. Goldstein, J. O. Gonzalez-Hernandez, S. Liuti, A. Rajan, arXiv: 1310.5157 [hep- 
ph].

[31] C. Lorce, arXiv:1401.7784 [hep-ph]. 